\title{
Which BMI for Diabetes Patients is Better? From the View of the Adipose Tissue Macrophage-Derived Exosome
}

\author{
Xiaojie Liu ${ }^{1-3}$ \\ Haichen Chu' \\ Yuzhi ji ${ }^{4}$ \\ Zeljko Bosnjak ${ }^{2}$ \\ Hushan $\mathrm{Ao}^{3, *}$ \\ Tianjun $\mathrm{Li}^{5, *}$ \\ 'Department of Anesthesiology, Affiliated \\ Hospital of Qingdao University, Qingdao, \\ Shandong Province, People's Republic of \\ China; ${ }^{2}$ Departments of Medicine and \\ Physiology, Medical College of Wisconsin, \\ Milwaukee, WI, USA; ${ }^{3}$ Department of \\ Anesthesiology, Fuwai Hospital, Chinese \\ Academy of Medical Sciences and Peking \\ Union Medical College, Beijing, People's \\ Republic of China; ${ }^{4}$ Obstetrics, Affiliated \\ Hospital of Qingdao University, Qingdao, \\ Shandong Province, People's Republic of \\ China; ${ }^{5}$ Department of Oncology, \\ Affiliated Hospital of Qingdao University, \\ Qingdao, Shandong Province, People's \\ Republic of China \\ *These authors contributed equally to \\ this work
}

Correspondence: Hushan Ao Department of Anesthesiology, Fuwai Hospital, Chinese Academy of Medical Sciences and Peking Union Medical College, No. 167 North Lishi Road, Xicheng District, Beijing, People's

Republic of China

Tel/Fax +86-10-68006210

Email aohushan@I26.com

Tianjun Li

Department of Oncology, Affiliated Hospital of Qingdao University, No. 59

Haier Road, Laoshan District, Qingdao,

Shandong Province, People's Republic of China

Tel/Fax +86-10-82913035

Email jxlitianjun@।63.com
Purpose: Diabetes, as a group of metabolic diseases, can elevate blood glucose, thus leading to the development of life-threatening complications. It is difficult to define the outcome for diabetics with different BMI. This review will illustrate the adipose tissue macrophagederived exosome in the diabetics with different BMI.

Patients and Methods: Insulin resistance in peripheral tissues can cause diabetes. The peripheral tissues include liver, muscle, or the adipose depots. Communication between these organs is fatal to the maintenance of glucose homeostasis. This review will illustrate this communication. Obesity is closely linked with diabetes. There are different changes in fat distribution in diabetic patients. Adipose tissue macrophages can secrete various hormones, including adiponectin, leptin, resistin and other classical cytokines, such as TNF- $\alpha$ and IL-6. Studies illustrated that exosomes from the adipose tissue, can modulate inter-organ cross-talk by regulating gene expression in other tissues.

Results: Adipose tissue macrophages exosomes links thin and fat individuals in the development of diabetes.

Conclusion: The molecular pathways initiated by exosomes such as miRNA in the situations of metabolic stress could help us gain a deeper knowledge of the pathophysiology of diabetes.

Keywords: BMI, obesity, macrophage, exosome, diabetes

\section{Introduction}

Obesity and T2D

Diabetes mellitus (DM) is the common metabolic disorders due to insufficiency in insulin secretion or action or both of them. The incidence of diabetes and its complications increases year by year. The international diabetes federation estimates that diabetes patients may rise to 629 million in $2045 .{ }^{1}$ It is now widely accepted that there are two primary types of diabetes, type 1 diabetes (T1D) caused by an autoimmune reaction. The body's immune system attacks the insulin-producing beta cells in the islets of the pancreas gland in T1D. Type 2 diabetes (T2D) is the result of an inadequate production of insulin. The insulin is insufficient in $\mathrm{T} 2 \mathrm{D}$, as the result of insulin resistance. ${ }^{2,3}$ Because aging population growing, obesity because of low physical activity, urbanization in developed countries, the prevalence of T2D has been increasing in the last few years and will continue to increase in the next few years. ${ }^{4-6}$

It is increasing of obesity over decades in both adults and children, and the number of severe or morbid obesity has increased to a greater extent than overweight and mild 
obesity recently. ${ }^{7}$ Body mass index (BMI), which is defined as weight in kilograms divided by height in meters squared. For adults, overweight is defined as BMI of 25.0 to $29.9 \mathrm{~kg}$ / $\mathrm{m} 2$. Obese is defined as BMI of $30 \mathrm{~kg} / \mathrm{m} 2$ or higher. ${ }^{8}$ Obesity is an established risk factor for metabolic and cardiovascular diseases and is defined as excessive lipid accumulation in the adipose tissue. ${ }^{9-11}$ Numerous adverse effects of overweight and obesity on health such as major cardiovascular $(\mathrm{CV})$ risk factors, including blood pressure, plasma lipids, glucose, inflammation, insulin resistance, all of which are the risk factors of T2D. ${ }^{12-14}$ Among them, insulin resistance is the most part of the process which can be illustrated in many studies (Figure 1). Our previous study found that compared with non-diabetic metabolic syndrome (MetS) patients, patients with diabetes had higher long-term major adverse cerebral cardiovascular events. Patients without MetS and diabetes were associated with lower incidence of long-term major adverse cerebral cardiovascular events after coronary artery bypass graft. ${ }^{15}$

Numerous studies illustrated that overweight or obesity can lead to higher mortality of cardiovascular disease. ${ }^{16,17}$ Meta-analysis also revealed that the risk for total mortality and hospitalization was highest in patients with chronic heart failure who were underweight defined as low BMI, whereas risk for cardiovascular mortality and hospitalization was lowest in overweight subjects. ${ }^{18}$ In the patients accepting carotid artery stenting or carotid endarterectomy for symptomatic carotid artery stenosis, BMI is not a periprocedural risk of stroke or death; however, overweight patients were associated with lower post procedural risk than that in normal weight group. ${ }^{19}$ Obesity was associated with lower risks in cardiac surgery, as showing a "U-shape" association between mortality and BMI classes which was observed in the cohort study including 557,720 patients. $^{20}$

Study showed that BMI $\geq 40.0$ was an independent risk factor for longer length of stay. Infection was a potential risk factor. $^{21}$ The other study also found underweight patients had the highest costs per patient while the obese and overweight patients had the lowest ones, which is presented as obesity paradox. ${ }^{22}$ Professor Del Prete concluded that 30 day mortality rates and early outcomes of obese patients who underwent $\mathrm{CABG}$ were similar to those of non-obese patients. ${ }^{23}$ For diabetes mellitus patients, professor Hällberg found that survival of diabetes mellitus patients deteriorated few years after operation when assessing the postoperative effects of metabolic syndrome and diabetes mellitus on the 16 year survival rate. ${ }^{24}$ Our previous study also found BMI of the diabetic patients undergoing $\mathrm{CABG}$ had no influence on the prognosis of survival and MACCEs. The gender differences in treatment may be important and affect the outcome in diabetes patients. ${ }^{25}$ For diabetes patients, which BMI is better is still a great question for us to study.
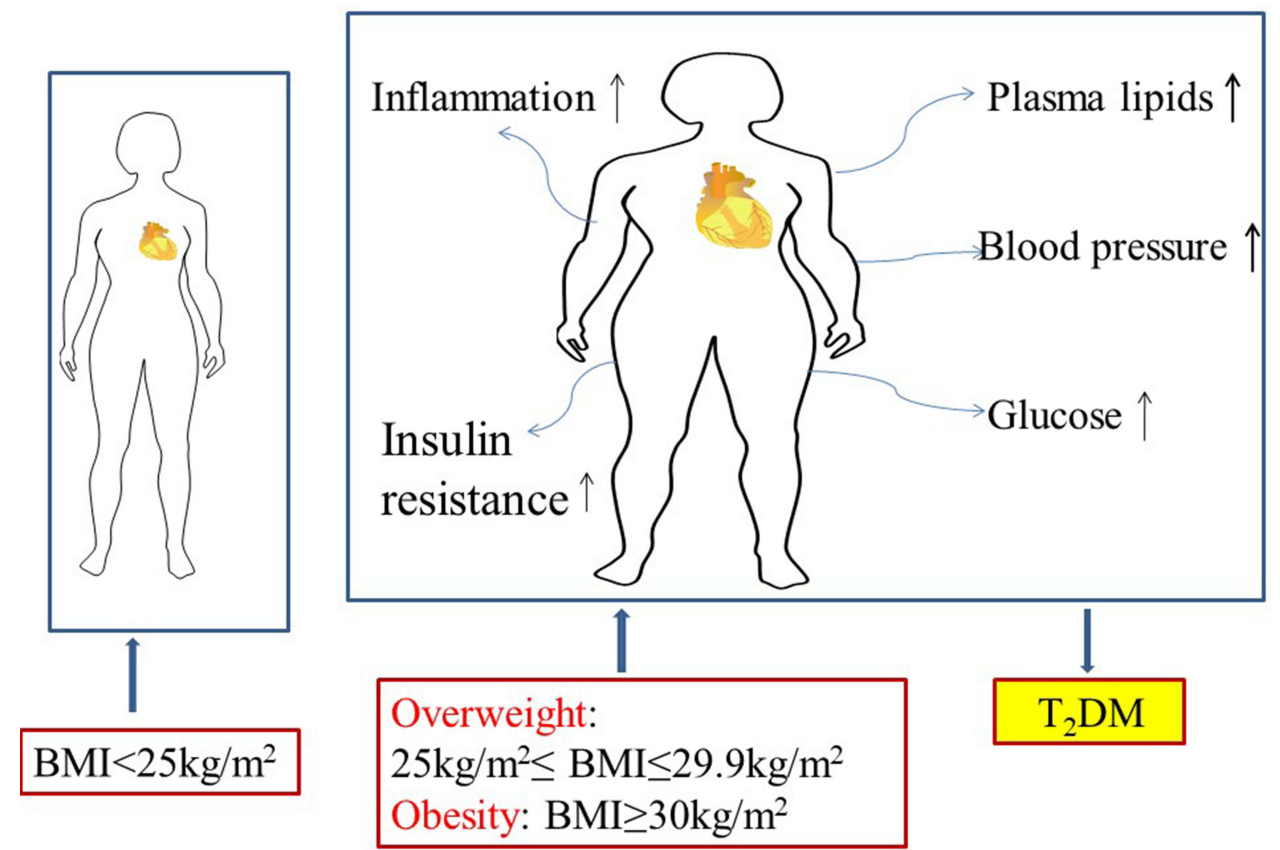

Figure I Adverse effects of overweight and obesity on health such as major cardiovascular (CV) risk factors, including blood pressure, plasma lipids, glucose, inflammation, insulin resistance. Overweight is defined as BMl of 25.0 to $29.9 \mathrm{~kg} / \mathrm{m} 2$. Obese is defined as BMI of $30 \mathrm{~kg} / \mathrm{m} 2$ or higher. 


\section{Insulin Resistance}

Reaven et al was first to provide a physiological mechanism for insulin resistance (IR), manifesting as hyperinsulinemia and found that it was the risk factor for the development of dyslipidemia, elevated blood pressure and glucose metabolism. ${ }^{26,27}$ IR is a systemic disorder affecting many organs by insulin-regulated pathways. The effects of insulin on target organs are different from the effects on traditional target organs. Insulin causes vasodilation by enhancing endothelial nitric oxide production by activating the phosphatidylinositol 3-kinase pathway. In insulin-resistant states, this pathway is impaired and the mitogen-activated protein kinase pathway further stimulates vasoconstriction. That is the mechanism of IR induced hypertension. The strong association of saltsensitive arterial hypertension with insulin resistance indicates the kidney participating in the insulin resistance syndrome. ${ }^{28}$

In addition to the kidney, insulin receptors also involve in the liver, skeletal muscle and white adipose tissue. In skeletal muscle, insulin promotes glucose utilization and storage with the help of increasing glucose transport and net glycogen synthesis. In liver, insulin activates glycogen synthesis by increasing lipogenic gene expression and decreasing gluconeogenic gene expression. Insulin suppresses lipolysis and increases glucose transport and lipogenesis in white adipocyte tissue (WAT), ${ }^{29,30}$ (Figure 2).

Recent studies illustrated that BMP4 prevented prevented obesity in adult mice by improving insulin sensitivity independent of weight reduction. The BMP antagonist Noggin was increased in WAT in obesity, while lack of brown adipocyte tissue. $^{31}$

However, the insulin resistance importance in the pathogenesis of T2D is strengthened by prospective studies that have revealed insulin resistance may become the best predictor of future T2D diagnosis. ${ }^{32}$

\section{Adipose Tissue and Hormones Secretion}

Adipose tissue is an organ, which can perform lots of significant physiological functions. Its excess in the body may result in pathological states in the organs and systems. Adipose tissue is different in both morphologically and physiologically. ${ }^{33}$ The human body adipose tissue can be divided into two main depots, subcutaneous adipose tissue (SAT) and visceral adipose tissue (VAT). VAT in turn can be further classified into intrathoracic, abdominal and so

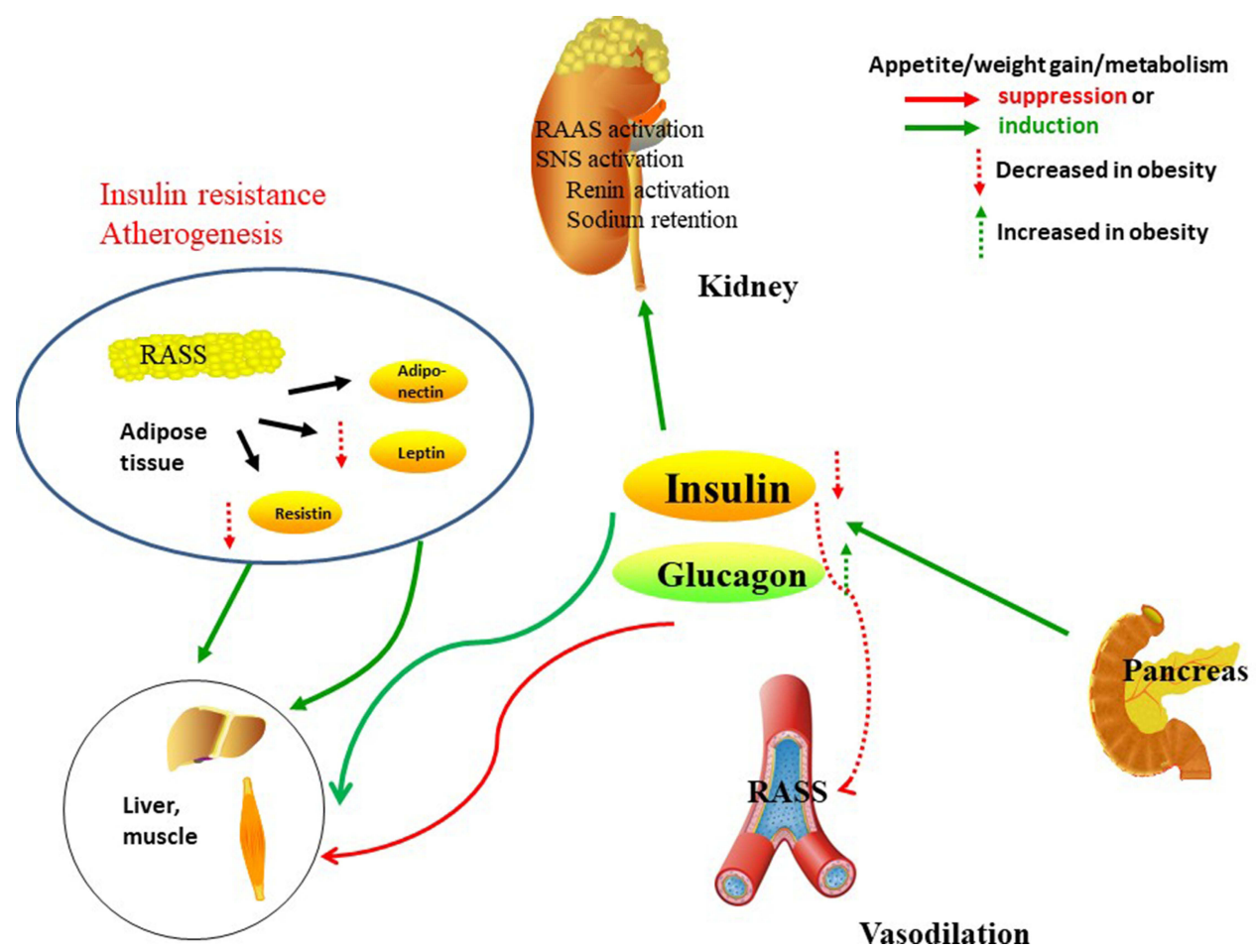

Figure 2 Insulin receptors in the kidney, liver, skeletal muscle and white adipose tissue. In skeletal muscle, insulin promotes glucose utilization and storage with the help of increasing glucose transport and net glycogen synthesis. In liver, insulin activates glycogen synthesis by increasing lipogenic gene expression and decreasing gluconeogenic gene expression. Insulin suppresses lipolysis and increases glucose transport and lipogenesis in white adipocyte tissue. In kidney, insulin participate in the RAAS, SNS activation, and the balance of sodium retention and renin activation. 
on. Intrathoracic adipose tissue can be classified into epicardial adipose tissue (EAT) and pericardial adipose tissue based on its location within or outside the human pericardium respectively. ${ }^{34,35}$ They include anatomical, cellular, molecular, clinical and prognostic differences. The adipocytes, with their endocrine function, lipolytic activity, can response to insulin and other hormones between subcutaneous adipose tissue (SAT) and visceral adipose tissue (VAT). Macrophages are more prevalent in visceral compared with subcutaneous fat. However, this was not found consistent by all researchers. ${ }^{36-38}$

Mammalian adipose tissue is traditionally divided into white and brown based on their function and morphology. White adipose tissue serves as energy storage while brown adipose tissue acts as the heat generator to maintain the core body temperature. ${ }^{39,40}$

Adipose tissue not only store fat but also play a protective role. It is an important endocrine organ which can generate and integrate signals to different tissues. It is also an enormously active endocrine organ, which can secrete various hormones, such as adiponectin, leptin, resistin and visfatin, in addition to the classical cytokines, including interleukin-6 (IL-6) and tumor necrosis factor-alpha (TNF- $\alpha$ ). Human epicardial adipose tissue (EAT) act locally on myocardium, atria and coronary arteries involving genes is omentin (ITLN1), which is the upregulated gene and secreted adipokine in EAT. Among EAT enriched genes, different patterns vary depending on adipose tissue distribution. We found a beige expression phenotype in EAT. Periventricular EAT highly expressed uncoupled protein 1 with genes overexpression in pericoronary.

EAT were implicated in proliferation, biosynthesis, and metabolism. Periatrial EAT expressed an atypical pattern with genes implicated in cardiac muscle contraction, and intracellular calcium signaling pathway. ${ }^{41}$

Previous studies illustrated that adipocytokine played significant roles in regulation of glucose, lipid and metabolism energy metabolism, which mediated insulin resistance in cardiovascular disease of type 2 diabetes. ${ }^{42,43}$ The other major adipokine except adipocytokine is leptin. Leptin levels increase in obesity. Subcutaneous fat has been a major source of circulating leptin levels. The effect of leptin is to inhibit appetite, enhance fatty acid oxidation, decrease glucose, stimulate thermogenesis, and reduce body weight. RBP4, chemerin, A-FABP, FGF21, fetuinA, myostatin, IL-6, are the other adipokines, all of which may play significant roles in insulin sensitivity. ${ }^{44,45}$ Gut microbiome and metabolomics are the potential future directions of new biological markers for measuring insulin resistance. Leptin/adiponectin and Angiotensin II imbalance may be important risk mediators of developing type 2 diabetes mellitus in cardiovascular diseases associated with abdominal obesity ${ }^{46}$ (Figure 3 ).

\section{The Inflammatory Response}

Obesity normally be associated with a state of chronic, low-grade inflammation. ${ }^{47}$ Being a chronic low-grade inflammatory state, it is associated with increased plasma levels of inflammatory markers. Weight reduction is associated with decrease in CRP level. For every $1 \mathrm{~kg}$ of weight loss, CRP levels dip by $0.13 \mathrm{mg} / \mathrm{L}{ }^{48}$ Exercise training is associated with a decrease in CRP levels regardless of the age or sex; however, greater improvements in CRP level occur with a decrease in BMI. ${ }^{49}$

Macrophages are the professional mononuclear phagocytes which can maintain tissue homeostasis and function by scavenging pathogens, debris, and apoptotic or necrotic cells. Circulating monocytes can differentiate into diverse resident macrophages in almost all tissues including liver (Kupffer cells), lung (alveolar macrophages), spleen, brain (microglia), bone (marrow macrophages) and fat (adipose tissue macrophages). Each macrophage phenotype has a specialized function and maintains the local tissue microenvironment. Macrophages can display heterogeneous phenotypes as demonstrated within adipose tissue macrophages (ATM).

Macrophages are immune cells in the adipose tissue. ${ }^{50}$ Following studies demonstrated the significant role of adipose tissue macrophages in metabolic disorder associated with obesity. ${ }^{51}$ Macrophages can secrete proinflammatory cytokines such as IL-6 and TNF-alpha. The macrophages and apoptotic cells co-culturing were found to increase pro-inflammatory cytokines such as IL-6, IL$1 \beta$, and MIP-2. ${ }^{52,53}$ However, several studies have shown that the apoptotic cells or phagocytosis do not induce inflammation. Human macrophage phagocytosed aged neutrophils without inflammatory responses. ${ }^{54}$

Obesity and diabetes can alter immune function and adipocyte size by several mechanisms. Adipokine can affect adipocyte size, with increase in adipocyte size and increase secretion of macrophage inflammatory protein $1 \beta$, IL-1RA, CCL2. ${ }^{55}$ Obesity is associated with adipocyte hypertrophy, with decrease in miRNAs in subcutaneous adipose tissue by attenuating CCL2 production. $^{56}$ Adipocytes release exosome-like vesicles taken up by monocytes where they promote differentiation into 


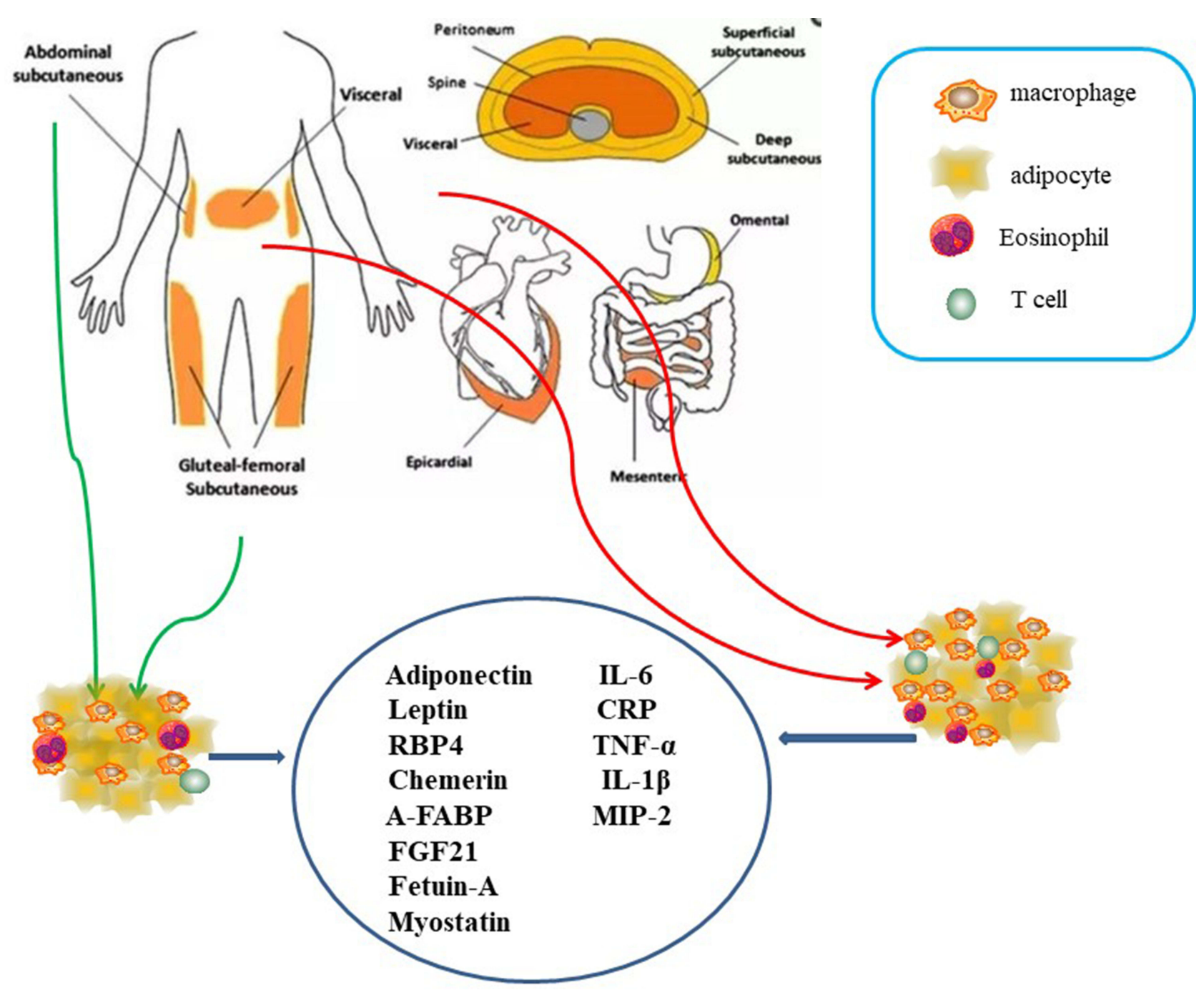

Figure 3 The human body adipose tissue can be divided into two main depots, subcutaneous adipose tissue (SAT) and visceral adipose tissue (VAT). VAT in turn can be further classified into intrathoracic, abdominal and so on. Intrathoracic adipose tissue can be classified into epicardial adipose tissue (EAT) and pericardial adipose tissue based on its location within or outside the human pericardium respectively. Adipocytokine played significant roles in regulation of glucose, lipid and metabolism energy metabolism, which mediated insulin resistance in cardiovascular disease of type 2 diabetes including leptin. RBP4, chemerin, A-FABP, FGF2I, fetuin-A, myostatin, IL-6, are the other adipokines, all of which may play significant roles in insulin sensitivity.

inflammatory macrophages and promote insulin resistance. ${ }^{57}$ Adipocytes and hepatocytes can secrete retinol binding protein 4 (RBP4), increased in obese and insulin resistant subjects by inducing proinflammatory cytokines (TNF- $\alpha$, IL-6, CCL-2, IFN- $\gamma$, GM-CSF) through JNK and TLR-4 pathways in macrophages. ${ }^{58}$ Activation of TLR4 signaling pathway requires mineralocorticoid receptors in bone marrow macrophages. ${ }^{59}$

\section{Adipose Tissue Macrophages (ATMs)}

Macrophages (M0) were differentiated by human peripheral blood monocytes and then polarized to M1 and M2 phenotypes by using LPS/IFN- $\gamma$ and IL-4/IL-13 respectively. $^{60}$
"M0" macrophages were originated from bone marrowderived monocytes. ' $\mathrm{M} 0$ ' macrophages can differentiate into several subsets depending on the stimuli. Lipopolysaccharide (LPS) can stimulate "M1" macrophages, expressed interferon (IFN)-tumor necrosis factor a (TNF-a) in vitro experiments, displaying the marker CD11c besides F4/80 and CD11b, by producing proinflammatory mediators like IL-6, IL-1 $\beta$, TNF-a, and nitric oxide (NO). "M2" macrophages possess anti-inflammatory phenotype and glucocorticoids stimulation by expressing the cell-surface markers CD11b, F4/80, CD301 and CD206, and secreting IL-4, IL-10 and IL-1 receptor antagonist (IL-1Ra).

M2 macrophages can be divided into three major variants by different stimuli. M2a was elicited by IL-13 or IL-4. 
M2b was obtained by triggering Fc gamma receptors in the presence of a Toll receptor. M2c was elicited by IL-10, TGFb or glucocorticoids. In obese patients, metabolic factors (eg high insulin, high glucose, oxidized phospholipids, free fatty acids, oxidized LDL give rise to a population of metabolic activated or oxidized macrophages ${ }^{61}$ (Figure 4).

Macrophages are essential to the innate immune response to pathogens. ${ }^{62}$ As antigen presentation cells, they regulate $\mathrm{T}$ cells of adipose tissue. Macrophages were the predominant immune cell type in obese adipose tissue, accounting for $30-50 \%$ of the nonadipocyte cell fraction. ${ }^{63,64}$ Greater number of macrophages in VAT than in SAT in both mice and humans, not seen in all studies. ${ }^{65-69}$ Visceral adipose cell had higher macrophage counts than abdominal subcutaneous adipose cell in gestational diabetes mellitus (GDM) pregnancies during cesarean delivery.

In the development of diabetes, macrophages contribute to the development of diabetic neuropathy. CD11b-positive microglia/macrophages gradually grow over the 28 days of testing after streptozocin injection which could cause diabetes. Flow cytometry showed that the infiltration of peripheral macrophages began to increase in 2 weeks $(\mathrm{P}<0.001)$ and reached a maximum at 4 weeks. ${ }^{70}$ Studies reported that in diabetic nephropathy macrophage cyclooxygenase-2 $(\mathrm{COX}-2)$ played a role in polarization and maintenance of a macrophage tissue-reparative M2 phenotype. $^{71}$

Adipocytes are important in the control of macrophage phenotype in adipose tissue. ${ }^{72,73}$ In healthy individuals, adiponectin secreted by adipocytes stimulates inducing M2-like polarization, suppression of ROS and ROSrelated genes. ${ }^{74}$ In obesity, decreased adiponectin production may favor prevalence of M1-like polarized macrophages. $^{75,76} \mathrm{M} 1$ macrophages were upregulated by glucose transporter GLUT1 that enhanced glucose consumption. ${ }^{77,78}$ ROS, produced in diabetes is increased in both endothelial cells and monocytes/macrophages which can result in activation of pro-inflammatory pathways and macrophage/endothelial cell interactions. ${ }^{79}$ Activated endothelial cells can increase the expression of adhesion molecules such as vascular cell adhesion molecule (VCAM) and intercellular adhesion molecule (ICAM); TNF- $\alpha$ and macrophage colony stimulating factor (MCSF), and macrophage chemoattractant protein-1 (MCP-1). ${ }^{80}$ Upregulation of adhesion molecules and chemokines promotes macrophage recruitment to the endothelium and their transmigration which maintains the

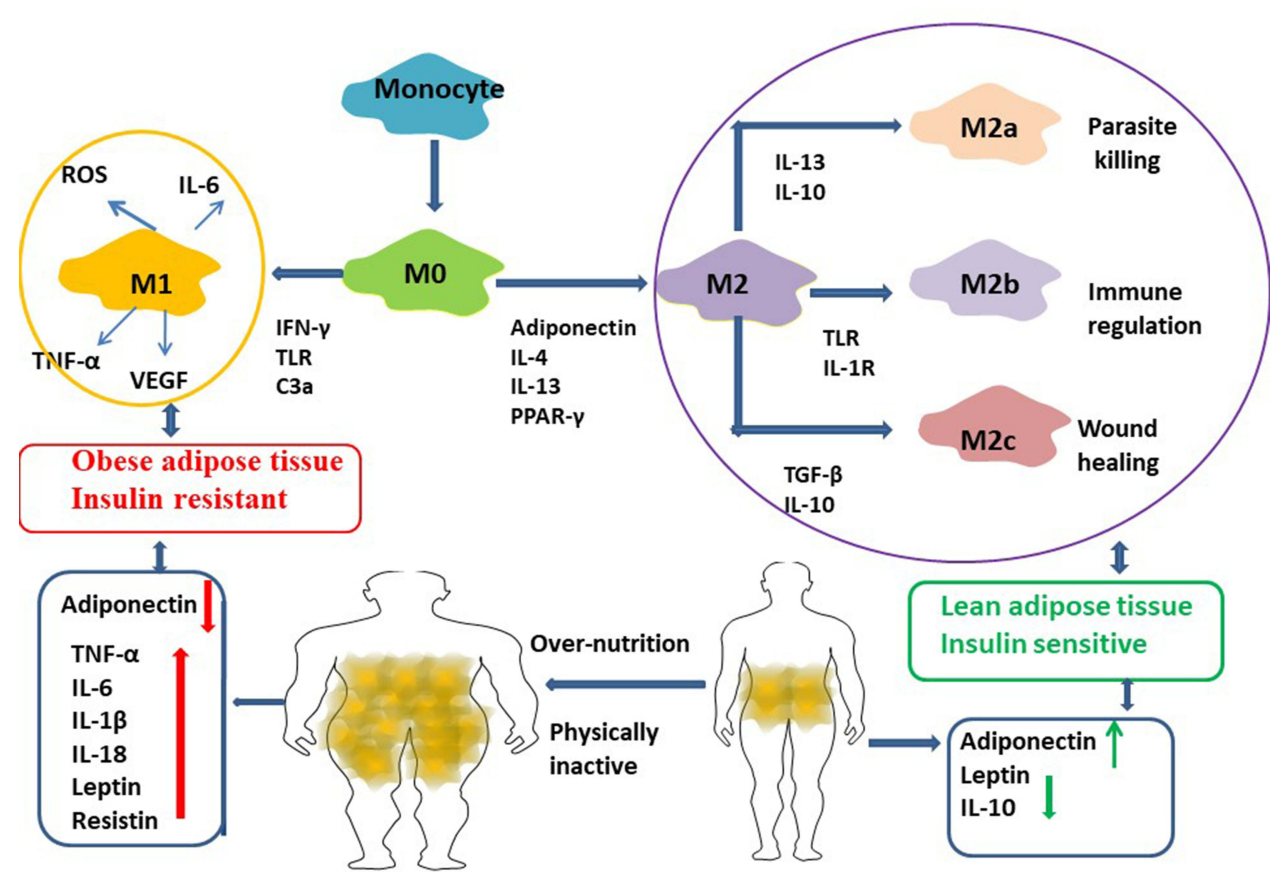

Figure 4 Macrophages (M0) were differentiated by human peripheral blood monocytes and then polarized to MI and M2 phenotypes by using LPS/IFN- $\gamma$ and IL-4/IL-I3 respectively. Macrophages from lean adipose tissue are M2 phenotype, whereas in obese adipose tissue macrophages is $\mathrm{MI}$ phenotype, expressing $\mathrm{F} 4 / 80+\mathrm{CDI}$ Ic+ and form crown-like structures (CLS) surrounding the adipocytes. MI macrophages mediate the metabolic complications, both in adipose tissue and by infiltration into other metabolic organs such as skeletal muscle. M2 macrophages can be divided into three major variants by different stimuli. M2a was elicited by IL-I 3 or IL-4. M2b was obtained by triggering Fc gamma receptors in the presence of a Toll receptor. M2c was elicited by IL-10, TGF-b or glucocorticoids. M2 macrophages express lower levels of inflammatory cytokines and higher levels anti-inflammatory cytokines. It participate in the lean adipose tissue insulin sensitive by the regulation of adiponectin, leptin and II10. 
inflammation in diabetic vascular dysfunction. Macrophage infiltration in diabetic nephropathy of $\mathrm{db} / \mathrm{db}$ mice correlated with expression of MCP-1, macrophage migration inhibitory factor (MIF) and M-CSF in glomeruli. $^{71}$

Upon activation, macrophages release cytokines and chemokines which initiate an inflammatory response. Tolllike receptors (TLR) are evolutionarily conserved pathogen-associated molecular pattern receptors. ${ }^{81}$

Macrophages in lean adipose tissue are M2 phenotype, whereas M1 phenotype is in obese adipose tissue, expressing F4/80+CD11c+ and form crown-like structures surrounding the adipocytes. ${ }^{82,83} \mathrm{M} 1$ macrophages mediate the metabolic complications, both in adipose tissue and by infiltration into other metabolic organs such as skeletal muscle. ${ }^{84}$ In mice, obesity induces M2 to M1 phenotype. M1 macrophages induce IR by inflammatory mediators, such as TNF- $\alpha$, IL-6 and nitric oxide. M2 macrophages maintain insulin sensitivity by IL-10 and activator of transcription 3 (STAT3) $^{61}$ (Figure 4).

\section{Exosomes and Exosomal miRNAs}

Exosomes are endosome-derived organelles (50-100nm) which are actively secreted through an exocytosis pathway. Recent studies have demonstrated that exosomes can mediate intercellular cross-talk under both in normal and pathological conditions. ${ }^{85,86}$ Although communication between adipose tissue and immune cells appears to be important in the interconnection between obesity and inflammation with the development of diabetes, research into the signals underlying this communication has, for the most part, been limited to analysis of the roles of cytokines and chemokines. Exosomes from adipose tissue of ob/ob mice can induce macrophage activation in a TLR4dependent manner and that the RBP4 incorporated in these exosomes plays a role in the induction of macrophage activation. ${ }^{87}$

Previous study found that isolated exosomes from adipose tissue of leptin-deficient ob/ob mice was injected into C57BL/6j wild type male mice and fed a high fat diet for 3 months. Exosomes obtained from the ob/ob mice can induce circulating levels of TNF $\alpha$ and IL- 6 and increased monocyte activation compared with exosomes from wild type mice. Injection of exosomes into wild-type C57BL/6 mice can result in the development of insulin resistance. When the exosomes were intravenously injected into TLR4 knockout B6 mice, the levels of glucose intolerance and insulin resistance were lower. RBP4 is enriched in the exosomes. Bone marrow-derived macrophages preincubated with recombinant RBP4 can lead to the attenuation of exosomes mediated induction of IL-6 and TNF- $\alpha^{87}$ (Figure 5). Exosomes from ob/ob mice also increased macrophage tissue infiltration and thus impaired insulin signaling. Similar results examining differences in exosomes isolated from obese and lean mice have also been described. $^{88,89}$

The possibility that adipose tissue-derived exosome-like vesicles are involved in this process and act as a mode of systemic communication has not been explored to any great extent. The biogenesis of exosomes is considered to be initiated in endosomes, and it is conceivable that the proteins contained in exosomes may influence the effects of the exosomes on the cells in terms of the type of response they elicit and the magnitude of the response. Exosomes contain

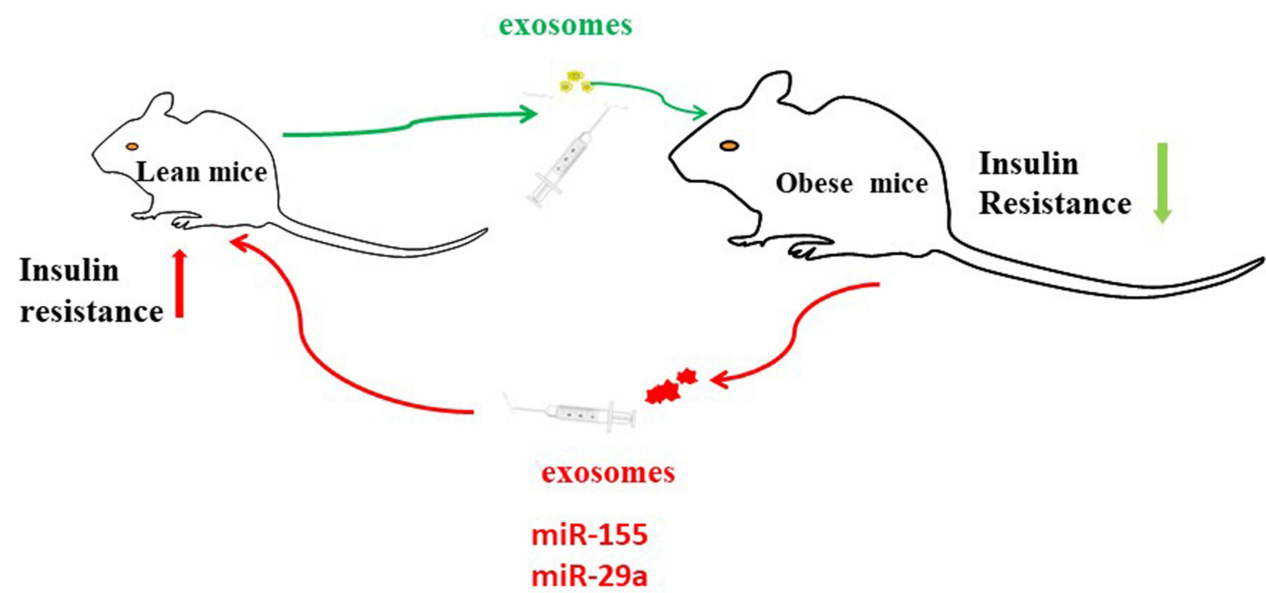

Figure 5 MiR-I55 is one of the miRNAs overexpressed in obese ATM Exos, miR-29a increased in obese ATMs derived exosomes. Administration of obese ATMs-Exos impairs insulin sensitivity of lean mice. 
molecular cargo including RNAs (mRNA, microRNAs and long noncoding RNAs), DNA. ${ }^{90-94}$

Studies have found that BMI correlates with exosomes concentration in pregnant women and aging cohort. ${ }^{95,96}$ Furthermore, significant higher levels of exosomes (measured subtypes included TMP, platelet-derived microparticles (PMP), P-selection+, endothelial cells microparticles (EMP), leukocyte-derived MPs (LMP), and tissue factor $\left.(\mathrm{TF})^{+}\right)$were found in obese individuals compared to normal weight controls. ${ }^{97}$ In addition, procoagulant activity was measured the clotting time of the exosomes. Which was significantly shorter for obese individuals compared to controls, indicating a higher procoagulant activity of exosomes from obese individuals. After a one year follow up period, the obese individuals who lost weight through diet/ exercise or through bariatric surgery had no significant change in exosomes levels compared to baseline levels. ${ }^{98}$ This contrasts with a previous report that showed that weight loss reduced exosomes levels. ${ }^{99}$ And exosomes participate in cell-to-cell communication in obesity. ${ }^{100}$

MicroRNAs (miRNA) are small non-coding RNA molecules that regulate gene expression by binding to messenger RNA. MiRNA-155 is released by macrophages in response to danger signals such as TLR ligands and LPS. ${ }^{101}$ MiRNA155 represses SOCS leading to JAK signaling and increase inflammation. Pro-inflammatory miRNA from macrophages are balanced by anti-inflammatory cytokines such as protectin. Protectin is synthesized by M2 macrophages and is involved in the resolution of inflammation and tissue healing. ${ }^{102}$ M2 macrophages express lower levels of inflammatory cytokines and higher levels anti-inflammatory cytokines. M2 macrophages also secrete transforming growth factor (TGF)- $\beta$, which promotes collagen expression and fibrosis but also have a crucial role in tissue repair ${ }^{103}$ (Figure 4). Treatment of lean mice with exosomes isolated from obese one could induce glucose intolerance and insulin resistance. Moreover, administration of control exosomes transfected with obesity-associated miRNA mimics strongly induces glucose intolerance in lean mice and results in central obesity and hepatic steatosis. This study found several miRNAs that may mediate these effects including miR-122, miR-192, miR-27a-3p, and miR-27b-3p. ${ }^{104}$

MiR-127 is an important regulator in the determination of macrophage phenotype in vivo and in vitro, regulating the key mechanisms that orchestrate protective immunity and inflammation. This miRNA promotes the development of M1 macrophage profile and simultaneously represses the transcription of gene markers of M2 phenotype. ${ }^{105}$ Studies illustrated that overexpression of miR-127 in macrophages significantly reduced the production of LPS induced proinflammatory cytokines. ${ }^{106} \mathrm{M} 2$ macrophages induced by adipose-derived stem cells (ADSC) derived exosomes not only expressed high levels of tyrosine hydroxylase responsible for catecholamine release, but also promoted ADSC proliferation and lactate production by favoring WAT beiging and homeostasis in response to high fat challenge. Studies have found that ADSC derived exosomes attenuate diet-induced obesity and metabolic disorders in HFD-fed mice. Administration of ADSC derived exosomes led to a resistance to sustain weight gain in HFD-fed mice during the later period of intervention, which was independent of food intake. For both visceral and subcutaneous white fat pads, HFD feeding caused dramatic increases in the percentages of fat weight to body weight, whereas exosomes significantly decreased these percentages. ${ }^{107}$ This study indicate that these strategies may hold promise for future therapies to combat obesity.

Previous studies found that miR-143 has been reported to be significantly upregulated in the mesenteric fat of obese mice, and obesity-induced over expression of this miRNA has been associated with impairment of glucose homeostasis. ${ }^{108,109}$ MiR-143 was more abundant in exosomes from non-activated than LPS-activated macrophages, suggesting that a proinflammatory state reduces the concentration of it. Treatment with TNF- $\alpha$ can down regulate miR-143 expression in adipocytes, suggesting that obesity-associated inflammation could deregulate the expression of miR-143, which may be one of the mechanisms that impair TNF- $\alpha$-induced pre-adipocyte differentiation in obese subjects. ${ }^{110}$

Expression of miR-145 was found to be attenuated in the omental adipose tissue of obese patients and diabetics with greater Arf6 expression, illustrating the role of miR145 in regulating macrophage-mediated inflammation targeting Arf6. By reducing the expression of Arf6 and subsequent signal transduction via NF-kappa, miR-145 plays a role in inhibiting the secretion of inflammatory factor. MiR-145 might be one of the candidates for antiinflammatory treatment for metabolic diseases. ${ }^{111}$

\section{Adipose Tissue Macrophage-Derived Exosomal miRNAs Insulin Sensitivity}

Chronic inflammatory diseases such as insulin resistance, Type 2 diabetes, neurodegenerative diseases etc, are 
shown to be caused due to imbalanced activation states of macrophages. ${ }^{112}$

MicroRNAs which are transcriptional/posttranscriptional regulators of gene expression drive several pathophysiological processes including macrophage polarization. However, the functional role of microRNAs in regulating inflammation induced insulin resistance is ill defined. Studies observed that the expression of miR-712 reduced in macrophages exposed to LPS and IFN- $\gamma$. Ectopic expression of miR-712 in mouse macrophages impaired the expression of iNOS protein and secretion of pro-inflammatory cytokines such as TNF- $\alpha$, IL- 6 and IFN$\beta$, all of which in turn led to improved insulin stimulated glucose uptake in co-cultured L6 myoblasts. Studies identified that miR-712 targeted the $3^{\prime}$ UTR of a potent inflammatory gene LRRK2 and dampened the phosphorylation of $\mathrm{p} 38$ and ERK1/2 kinases. The regulatory role of miR712 can restore insulin stimulated glucose uptake by myoblasts through down-regulating macrophage mediated inflammatory responses. ${ }^{113}$

MiR-155 is one of the miRNAs overexpressed in obese ATM Exos, and earlier studies have shown that PPAR $\gamma$ is a miR-155 target. Following studies showed that miR-155 KO animals are insulin sensitive and glucose tolerant compared to controls. Furthermore, transplantation of WT bone marrow into miR-155KO mice mitigated this phenotype. ATMs secrete exosomes containing miRNA cargo. These miRNAs can be transferred to insulin target cell types by mechanisms of paracrine or endocrine regulation with effects on cellular insulin action, in vivo insulin sensitivity, and overall glucose homeostasis. ${ }^{89}$ Studies showed that miR-29a increased in obese ATMs derived exosomes (ATMs-Exos) and can be transferred into adipocytes, myocytes and hepatocytes causing insulin resistance in vitro and in vivo. Administration of obese ATMs-Exos impairs insulin sensitivity of lean mice. Knockdown miR-29a level in obese ATM-Exos blunts this effect. PPAR- $\delta$ is identified to function as downstream target of miR-29a in regulating insulin resistance. PPAR- $\delta$ agonist GW501516 partially rescued the insulin resistance induced by miR-29a ${ }^{114}$ (Figure 5).

\section{BMI and Abdominal Adipose Tissue}

Studies have found that women with impaired glucose metabolism (IGM) had higher BMI/fat mass. BMI was the best discriminator of normal glucose tolerance (NGT) versus IGM. Waist-to-height ratio and adipocyte volume were most strongly associated with HOMA-IR. ${ }^{115}$ Compared with BMI, abdominal adipose tissue was the novel cardiovascular risk biomarker. ${ }^{116}$ Another study illustrated that total macrophage numbers in subcutaneous adipose tissue increased with (BMI), with a similar increase seen in the proportion of phagocytic CD14 $+\mathrm{CD} 16+\mathrm{CD} 36$ high macrophages. There was an inverse correlation between anti-inflammatory CD14+CD16CD163+ macrophages and BMI. These correlations disappeared after excluding obese subjects (BMI $\geqslant 30 \mathrm{~kg} / \mathrm{m}^{2}$ ) from the analysis. None of these subpopulations were significantly related to BMI in visceral adipose tissue. Obesity per se is associated with distinct, highly phagocytic macrophage accumulation in human subcutaneous adipose tissue. $^{117}$

\section{Conclusion}

In summary, we showed that BMI and its prognosis in diabetes individuals. We found that the subtypes of macrophages and its relationship with different adipose tissue, especially the SAT and VAT. Different adipose depots contribute different macrophages and exosomal miRNAs to the circulation. We also showed that these adiposederived exosomal miRNAs can have systemic effects, including regulating of insulin resistance in lean and obesity. As a product of different adipose depots, these exosomal miRNAs could also change in level in diseases with altered fat mass, such as obesity, or altered adipose distribution and function, such as diabetes and aging. Which BMI is better for diabetes is not an easy question which covers the proportion of adipose tissue and it is not just the ratio of weight to height squared. Adipose-derived exosomal miRNAs can act as regulators of metabolism in distant tissues providing a new mechanism of cell-cell crosstalk.

\section{Patient and Public Involvement}

No patient involved.

\section{Data Sharing Statement}

All the data generated and/or analyzed during this study are included in this published article.

\section{Ethics Approval and Consent to Participate}

Not applicable. 


\section{Funding}

Youth Funding of the Affiliated Hospital of Qingdao University. The Award/Grant number is not applicable.

\section{Disclosure}

The authors declare that they have no competing interests.

\section{References}

1. International Diabetes Federation. IDF Diabetes Atlas. 9th ed. Diabetes Atlas; 2019. Available from: http://www.diabetesatlas. org/.

2. Buse JB, Wexler DJ, Tsapas A, et al. 2019 update to: management of hyperglycemia in type 2 diabetes, 2018. A consensus report by the American Diabetes Association (ADA) and the European Association for the Study of Diabetes (EASD). Diabetes Care. 2020;43(2):487-493. doi:10.2337/dci19-0066

3. American Diabetes Association. 2. Classification and diagnosis of diabetes: standards of medical care in diabetes-2020. Diabetes Care. 2020;43(Supplement_1):S14-S31. doi:10.2337/dc20-S002

4. Campbell MR. Review of current status of molecular diagnosis and characterization of monogenic diabetes mellitus: a focus on next-generation sequencing. Expert Rev Mol Diagn. 2020;20 (4):413-420. doi:10.1080/14737159.2020.1730179

5. Cai L, Wheeler E, Kerrison ND, et al. Genome-wide association analysis of type 2 diabetes in the EPIC-InterAct study. Sci Data. 2020;7(1):393. doi:10.1038/s41597-020-00716-7

6. Kiguli J, Helle Mölsted Alvessond, Roy William Mayega, et al. Dietary patterns and practices in rural eastern Uganda: Implications for prevention and management of type 2 diabetes. Appetite. 2019;143:104409. doi:10.1016/j.appet.2019.104409

7. Ge L, Sadeghirad B, Ball GDC, et al. Comparison of dietary macronutrient patterns of 14 popular named dietary programmes for weight and cardiovascular risk factor reduction in adults: systematic review and network meta-analysis of randomized trials. BMJ. 2020;369:m696. doi:10.1136/bmj.m696

8. Ward ZJ, Bleich SN, Cradock AL, et al. Projected U.S. state-level prevalence of adult obesity and severe obesity. $N$ Engl J Med. 2019;381(25):2440-2450. doi:10.1056/NEJMsa1909301

9. Cameron AJ, Magliano DJ, Söderberg S. A systematic review of the impact of including both waist and hip circumference in risk models for cardiovascular diseases, diabetes and mortality. $\mathrm{J} \mathrm{Am}$ Heart Assoc. 2020;9:e015189. doi:10.1161/JAHA.119.015189

10. Lyall DM, Celis-Morales C, Ward J, et al. Association of body mass index with cardiometabolic disease in the UK Biobank: a Mendelian randomization study. JAMA Cardiol. 2017;2 (8):882-889. doi:10.1001/jamacardio.2016.5804

11. Yusuf S, Joseph P, Rangarajan S, et al. Modifiable risk factors, cardiovascular disease, and mortality in 155722 individuals from 21 high-income, middle-income, and low-income countries (PURE): a prospective cohort study. Lancet. 2019;6736:32 008-32012.

12. Wang T, Lu J, Shi L, et al. Association of insulin resistance and $\beta$ cell dysfunction with incident diabetes among adults in China: a nationwide, population-based, prospective cohort study. Lancet Diabetes Endocrinol. 2020;8(2):115-124. doi:10.1016/S22138587(19)30425-5

13. Nakamura M, Sadoshima J. Cardiomyopathy in obesity, insulin resistance and diabetes. J Physiol. 2020;598(14):2977-2993. doi:10.1113/JP276747

14. Wu H, Ballantyne CM. Metabolic inflammation and insulin resistance in obesity. Circ Res. 2020;126(11):1549-1564. doi:10.1161/ CIRCRESAHA.119.315896
15. Wang L, Qian X, Wang M, et al. Which factor is the most effective one in metabolic sydrome on the outcomes after coronary artery bypass graft surgery? cohort study of 5 Years. $J$ Cardiothorac Surg. 2018;13(1):1. doi:10.1186/s13019-0170682-5

16. Collaborators, et al. Health effects of overweight and obesity in 195 countries over 25 years. $N$ Engl J Med. 2017;377(1):13-27. doi:10.1056/NEJMoa1614362

17. Klatsky AL, Zhang J, Udaltsova N, et al. Body mass index and mortality in a very large cohort: is it really healthier to be overweight? Perm J. 2017;21:16-142. doi:10.7812/TPP/16-142

18. Sharma A, Lavie CJ, Borer JS, et al. Meta-analysis of the relation of body mass index to all-cause and cardiovascular mortality and hospitalization in patients with chronic heart failure. $\mathrm{Am}$ $J$ Cardiol. 2015;115(10):1428-1434. doi:10.1016/j.amjcard.20 15.02 .024

19. Volkers EJ, Greving JP, Hendrikse J, et al. Body mass index and outcome after revascularization for symptomatic carotid artery stenosis. Neurology. 2017;88(21):2052-2060. doi:10.1212/WNL. 0000000000003957

20. Mariscalco G, Wozniak MJ, Dawson AG, et al. Body mass index and mortality among adults undergoing cardiac surgery: a nationwide study with a systematic review and meta-Analysis. Circulation. 2017;135(9):850-863. doi:10.1161/CIRCULATIONA HA. 116.022840

21. Terada T, Johnson JA, Norris C, et al. Severe obesity is associated with increased risk of early complications and extended length of stay following coronary artery bypass grafting surgery. $J$ Am Heart Assoc. 2016;5(6):e003282. doi:10.11 61/JAHA.116.003282

22. Johnson AP, Parlow JL, Milne B, et al. Economies of scale: body mass index and costs of cardiac surgery in Ontario, Canada. Eur $J$ Health Econ. 2017;18(4):471-479. doi:10.1007/s10198-0160803-4

23. Friedrich C, Berndt R, Haneya A, et al. Sex-specific outcome after minimally invasive direct coronary artery bypass for single-vessel disease. Interact Cardiovasc Thorac Surg. 2020;30 (3):380-387. doi:10.1093/icvts/ivz279

24. Hällberg V, Sundaram V, Wilson B, et al. Adverse events after coronary artery bypass grafting in patients with preoperative metabolic syndrome: a 10-year follow-up of the veterans affairs database. J Thorac Cardiovasc Surg. 2020;5223:32384-32389.

25. Liu X, Zhang W, Wang L, et al. Male patients with diabetes undergoing coronary artery bypass grafting have increased major adverse cerebral and cardiovascular events. Interact Cardiovasc Thorac Surg. 2019;28(4):607-612. doi:10.1093/ icvts/ivy 287

26. Luc K, Schramm-Luc A, Guzik TJ, et al. Oxidative stress and inflammatory markers in prediabetes and diabetes. J Physiol Pharmacol. 2019;70:1.

27. Reaven G. Metabolic syndrome: pathophysiology and implications for management of cardiovascular disease. Circulation. 2002;106(3):286-288. doi:10.1161/01.CIR.0000 019884.36724.D9

28. Artunc F, Schleicher E, Weigert C, et al. The impact of insulin resistance on the kidney and vasculature.Nat. Rev Nephrol. 2016;12(12):721-737. doi:10.1038/nrneph.2016.145

29. Petersen MC. Mechanisms of insulin action and insulin resistance. Physiol Rev. 2018;98(4):2133-2223. doi:10.1152/ physrev.00063.2017

30. Barinda AJ, Ikeda K, Nugroho DB, et al. Endothelial progeria induces adipose tissue senescence and impairs insulin sensitivity through senescence associated secretory phenotype. Nat Commun. 2020;11(1):481. doi:10.1038/s41467-020-14387-w

31. Tanaka M. Improving obesity and blood pressure. Hypertens Res. 2020;43(2):79-89. doi:10.1038/s41440-019-0348-x 
32. Hoffmann JM, Grünberg JR, Hammarstedt A, et al. BMP4 gene therapy enhances insulin sensitivity but not adipose tissue browning in obese mice. Mol Metab. 2020;32:15-26. doi:10.1016/j. molmet.2019.11.016

33. Oikonomou EK, Antoniades C. The role of adipose tissue in cardiovascular health and disease. Nat Rev Cardiol. 2019;16:83-99. doi:10.1038/s41569-018-0097-6

34. Bendor CD, Bardugo A, Pinhas-Hamiel O, et al. Cardiovascular morbidity, diabetes and cancer risk among children and adolescents with severe obesity. Cardiovasc Diabetol. 2020;19(1):79. doi:10.1186/s12933-020-01052-1

35. Iacobellis G. Local and systemic effects of the multifaceted epicardial adipose tissue depot. Nat Rev Endocrinol. 2015;11 (6):363-371. doi:10.1038/nrendo.2015.58

36. Vijay J, Gauthier MF, Biswell RL, et al. Single-cell analysis of human adipose tissue identifies depot and disease specific cell types. Nat Metab. 2020;2(1):97-109. doi:10.1038/s42255-0190152-6

37. Poledne R, Malinska H, Kubatova H, et al. Polarization of macrophages in human adipose tissue is related to the fatty acid spectrum in membrane phospholipids. Nutrients. 2019;12(1):8. doi:10.3390/nu12010008

38. Petrus P, Lecoutre S, Dollet L, et al. Glutamine links obesity to inflammation in human white adipose tissue. Cell Metab. 2020;31 (2):375-390.e11. doi:10.1016/j.cmet.2019.11.019

39. Kaisanlahti A, Glumoff T. Browning of white fat: agents and implications for beige adipose tissue to type 2 diabetes. J Physiol Biochem. 2019;75(1):1-10. doi:10.1007/s13105-0180658-5

40. Wang W, Seale P. Control of brown and beige fat development. Nat Rev Mol Cell Biol. 2016;17(11):691-702. doi:10.1038/ nrm.2016.96

41. Gaborit B, Venteclef N, Ancel P, et al. Human epicardial adipose tissue has a specific transcriptomic signature depending on its anatomical peri-atrial, peri-ventricular, or peri-coronary location. Cardiovasc Res. 2015;108(1):62-73. doi:10.1093/cvr/ cvv208

42. Jaganathan R, Ravindran R, Dhanasekaran S, et al. Emerging role of adipocytokines in type 2 diabetes as mediators of insulin resistance and cardiovascular Disease. Can J Diabetes. 2018;42 (4):446-456. doi:10.1016/j.jcjd.2017.10.040

43. Akkus G, Evran M, Sert M, et al. Adipocytokines in non-functional adrenal incidentalomas and relation with insulin resistance parameters. Endocr Metab Immune Disord Drug Targets. 2019;19(3):326-332. doi:10.2174/1871530318666181 009112042

44. Noordam R, Boersma V, Verkouter I, et al. The role of C-reactive protein, adiponectin and leptin in the association between abdominal adiposity and insulin resistance in middle-aged individuals Nutr Metab Cardiovasc Dis. 2020;30(8):1306-1314. doi:10.1016/ j.numecd.2020.04.021

45. Park SE, Park C-Y, Sweeney G, et al. Biomarkers of insulin sensitivity and insulin resistance: past, present and future. Crit Rev Clin Lab Sci. 2015;52(4):180-190. doi:10.3109/104083 63.2015.1023429

46. Carmen Zaha D, Vesa C, Uivarosan D, et al. Influence of inflammation and adipocyte biochemical markers on the components of metabolic syndrome. Exp Ther Med. 2020;20(1):121-128. doi:10.3892/etm.2020.8663

47. Pantham P, Aye IL, Powell TL, et al. Inflammation in maternal obesity and gestational diabetes mellitus. Placenta. 2015;36 (7):709-715. doi:10.1016/j.placenta.2015.04.006

48. Aasbrenn M, Farup PG, Videm V, et al. Changes in C-reactive protein, neopterin and lactoferrin differ after conservative and surgical weight loss in individuals with morbid obesity. Sci Rep. 2019;9(1):17695. doi:10.1038/s41598-019-54107-z
49. Fedewa MV, Hathaway ED, Ward-Ritacco CL, et al. Effect of exercise training on $\mathrm{C}$ reactive protein: a systematic review and meta-analysis of randomised and non-randomised controlled trials. Br J Sports Med. 2017;51(8):670-676. doi:10.1136/ bjsports-2016-095999

50. Wang P, Loh KH, Wu M, et al. A leptin-BDNF pathway regulating sympathetic innervation of adipose tissue. Nature. 2020;583 (7818):839-844. doi:10.1038/s41586-020-2527-y

51. Tsuhako R, Yoshida H, Sugita $C$, et al. Naringenin suppresses neutrophil infiltration into adipose tissue in high-fat diet-induced obese mice. J Nat Med. 2020;74(1):229-237. doi:10.1007/ s11418-019-01332-5

52. Medina CB, Mehrotra P, Arandjelovic S, et al. Metabolites released from apoptotic cells act as tissue messengers. Nature. 2020;580(7801):130-135. doi:10.1038/s41586-020-2121-3

53. Festuccia WT. Macrophage and adipocyte mitochondrial dysfunction in obesity-induced metabolic diseases. Mol Nutr Food Res. 2020;e1900768. doi:10.1002/mnfr.201900768

54. Kanoke A, Nishijima Y, Ljungberg M, et al. The effect of type 2 diabetes on CD36 expression and the uptake of oxLDL: diabetes affects CD36 and oxLDL uptake. Exp Neurol. 2020;334:113461. doi:10.1016/j.expneurol.2020.113461

55. Skurk T, Alberti-Huber C, Herder C, et al. Relationship between adipocyte size and adipokine expression and secretion. J Clin Endocrinol Metab. 2007;92(3):1023-1033. doi:10.1210/jc.2006-1055

56. Arner E, Mejhert N, Kulyté A, et al. Adipose tissue microRNAs as regulators of CCL2 production in human obesity. Diabetes. 2012;61(8):1986-1993. doi:10.2337/db11-1508

57. Ji C, Guo X. The clinical potential of circulating microRNAs in obesity. Nat Rev Endocrinol. 2019;15(12):731-743. doi:10.1038/ s41574-019-0260-0

58. Norseen J, Hosooka T, Hammarstedt A, et al. Retinol-binding protein 4 inhibits insulin signaling in adipocytes by inducing proinflammatory cytokines in macrophages through a c-Jun $\mathrm{N}$ terminal kinase- and toll-like receptor 4-dependent and retinol-independent mechanism. Mol Cell Biol. 2012;32 (10):2010-2019. doi:10.1128/MCB.06193-11

59. Shen JZ, Morgan J, Tesch GH, et al. Cardiac tissue injury and remodeling is dependent upon MR regulation of activation pathways in cardiac tissue macrophages. Endocrinology. 2016;157 (8):3213-3223. doi:10.1210/en.2016-1040

60. Tarique AA, Logan J, Thomas E, et al. Phenotypic, functional, and plasticity features of classical and alternatively activated human macrophages. Am J Respir Cell Mol Biol. 2015;53 (5):676-688. doi:10.1165/rcmb.2015-0012OC

61. Russo L, Lumeng CN. Properties and functions of adipose tissue macrophages in obesity. Immunology. 2018;155(4):407-417. doi:10.1111/imm.13002

62. Burhans MS, Hagman DK, Kuzma JN, et al. Contribution of adipose tissue inflammation to the development of type 2 diabetes mellitus. Compr Physiol. 2018;9(1):1-58. doi:10.1002/cphy.c170040

63. Zatterale F, Longo M, Naderi J, et al. Chronic adipose tissue inflammation linking obesity to insulin resistance and type 2 diabetes. Front Physiol. 2020;10:1607. doi:10.3389/fphys.2019.01607

64. Brooks-Worrell BM, Palmer JP. Setting the stage for islet autoimmunity in type 2 diabetes: obesity-associated chronic systemic inflammation and endoplasmic reticulum (ER) Stress. Diabetes Care. 2019;42(12):2338-2346. doi:10.2337/dc19-0475

65. Altintas MM, Azad A, Nayer B, et al. Mast cells, macrophages, and crown-like structures distinguish subcutaneous from visceral fat in mice. J Lipid Res. 2011;52(3):480-488. doi:10.1194/jlr.M011338

66. González-Granillo M, Savva C, Li X, et al. Selective estrogen receptor (ER) $\beta$ activation provokes a redistribution of fat mass and modifies hepatic triglyceride composition in obese male mice. Mol Cell Endocrinol. 2020;502:110672. doi:10.1016/j.mce.20 19.110672 
67. Peleli M, Ferreira DMS, Tarnawski L, et al. Dietary nitrate attenuates high-fat diet-induced obesity via mechanisms involving higher adipocyte respiration and alterations in inflammatory status. Redox Biol. 2020;28:101387. doi:10.1016/j.redox.2019. 101387

68. Cancello R, Tordjman J, Poitou C, et al. Increased infiltration of macrophages in omental adipose tissue is associated with marked hepatic lesions in morbid human obesity. Diabetes. 2006;55 (6):1554-1561. doi:10.2337/db06-0133

69. Harlev A, Aricha-Tamir B, Shaco-Levy R, et al. Macrophage infiltration and stress-signaling in omental and subcutaneous adipose tissue in diabetic pregnancies. J Matern Fetal Neonatal Med. 2014;27(12):1189-1194. doi:10.3109/14767058.2013.853734

70. Harman-Boehm I, Bluher M, Redel H, et al. Macrophage infiltration into omental versus subcutaneous fat across different populations: effect of regional adiposity and the comorbidities of obesity. J Clin Endocrinol Metab. 2007;92(6):2240-2247. doi:10. 1210/jc.2006-1811

71. Wang X, Yao B, Wang Y, et al. Macrophage cyclooxygenase-2 protects against development of diabetic nephropathy. Diabetes. 2017;66(2):494-504. doi:10.2337/db16-0773

72. Lihn AS. Adiponectin: action, regulation and association to insulin sensitivity. Obes Rev. 2015;2015:13-21.

73. Ouchi N, Higuchi A, Ohashi $K$, et al. Sfrp5 is an anti-inflammatory adipokine that modulates metabolic dysfunction in obesity. Science. 2010;329(5990):454-457. doi:10.1126/ science. 1188280

74. Zhu Q, An YA, Kim M, et al. Suppressing adipocyte inflammation promotes insulin resistance in mice. Mol Metab. 2020;39:101010. doi:10.1016/j.molmet.2020.101010

75. Goh J, Goh KP, Abbasi A, et al. Adipose tissue macrophages: new frontiers in obesity research?. Front Endocrinol. 2016;7:65.

76. Martinez B, Soñanez-Organis JG, Viscarra JA, et al. Glucose delays the insulin-induced increase in thyroid hormone-mediated signaling in adipose of prolong-fasted elephant seal pups. Am J Physiol Regul Integr Comp Physiol. 2016;310(6):R502-12. doi:10.1152/ajpregu.00054.2015

77. Nishizawa T, Kanter JE, Kramer F, et al. Testing the role of myeloid cell glucose flux in inflammation and atherosclerosis. Cell Rep. 2014;7:356-365.

78. Freemerman AJ, Johnson AR, Sacks GN, et al. Metabolic reprogramming of macrophages: glucose transporter 1 (GLUT1)-mediated glucose metabolism drives a proinflammatory phenotype. J Biol Chem. 2014;289(11):7884-7896. doi:10.1074/ jbc.M113.522037

79. Giacco F, Brownlee M. Oxidative stress and diabetic complications. Circ Res. 2010;107(9):1058-1070. doi:10.1161/ CIRCRESAHA.110.223545

80. Tabit CE, et al. Endothelial dysfunction in diabetes mellitus: molecular mechanisms and clinical implications. Rev Endocr Metab Disord. 2020;11:61-74.

81. Sun JJ, Tang L, Zhao X-P, et al. Infiltration of blood-derived macrophages contributes to the development of diabetic neuropathy. J Immunol Res. 2019;2019:7597382. doi:10.1155/ 2019/7597382

82. Ferrante AW Jr. Obesity-induced inflammation: a metabolic dialogue in the language of inflammation. J Intern Med. 2007;262 (4):408-414. doi:10.1111/j.1365-2796.2007.01852.x

83. Gericke M, Weyer U, Braune J, et al. A method for long-term live imaging of tissue macrophages in adipose tissue explants. Am $J$ Physiol Endocrinol Metab. 2015;308(11):E1023-E1033. doi:10.1152/ajpendo.00075.2015

84. Samaan MC, Marcinko K, Sikkema S, et al. Endurance interval training in obese mice reduces muscle inflammation and macrophage content independently of weight loss. Physiol Rep. 2014;2 (5):e12012. doi:10.14814/phy2.12012
85. Toh WS, Lai RC, Zhang B, et al. MSC exosome works through a protein-based mechanism of action. Biochem Soc Trans. 2018;46 (4):843-853. doi:10.1042/BST20180079

86. Valadi H, Ekström K, Bossios A, et al. Exosome-mediated transfer of mRNAs and microRNAs is a novel mechanism of genetic exchange between cells. Nat Cell Biol. 2007;9(6):654-659. doi:10.1038/ncb1596

87. Deng ZB, Poliakov A, Hardy RW, et al. Adipose tissue exosome-like vesicles mediate activation of macrophage-induced insulin resistance. Diabetes. 2009;58(11):2498-2505. doi:10.23 37/db09-0216

88. Martinez MC, Andriantsitohaina R. Extracellular vesicles in metabolic syndrome. Circ Res. 2017;120(10):1674-1686. doi:10.1161/CIRCRESAHA.117.309419

89. Ying W, Riopel M, Bandyopadhyay G, et al. Adipose tissue macrophage derived exosomal miRNAs can modulate in vivo and in vitro insulin sensitivity. Cell. 2017;171(2):372-384. doi:10.1016/j.cell.2017.08.035

90. Greening DW, Xu R, Gopal SK, et al. Proteomic insights into extracellular vesicle biology defining exosomes and shed microvesicles. Expert Rev Proteomics. 2017;14(1):69-95. doi:10.1080/14789450.2017.1260450

91. Kahlert C, Melo SA, Protopopov A, et al. Identification of double-stranded genomic DNA spanning all chromosomes with mutated KRAS and p53 DNA in the serum exosomes of patients with pancreatic cancer. J Biol Chem. 2014;289(7):3869-3875. doi:10.1074/jbc.C113.532267

92. Kim KM, Abdelmohsen K, Mustapic M, et al. RNA in extracellular vesicles. Wiley Interdiscip Rev RNA. 2017;8(4):10. doi:10.1002/wrna.1413

93. Thakur BK, Zhang H, Becker A, et al. Double-stranded DNA in exosomes: a novel biomarker in cancer detection. Cell Res. 2014;24(6):766-769. doi:10.1038/cr.2014.44

94. Yanez-Mo M, Siljander PRM, Andreu Z, et al. Biological properties of extracellular vesicles and their physiological functions. J Extracell Vesicles. 2015;4(1):27066. doi:10.3402/ jev.v4.27066

95. Elfeky O, Longo S, Lai A, et al. Influence of maternal BMI on the exosomal profile during gestation and their role on maternal systemic inflammation. Placenta. 2017;50:60-69. doi:10.1016/j. placenta.2016.12.020

96. Eitan E, Green J, Bodogai M, et al. Age-related changes in plasma extracellular vesicle characteristics and internalization by leukocytes. Sci Rep. 2017;7(1):1342. doi:10.1038/s41598-01701386-z

97. Campello E, Zabeo E, Radu CM, et al. Hypercoagulability in overweight and obese subjects who are asymptomatic for thrombotic events. Thromb Haemost. 2015;113(01):85-96. doi:10.1160/TH1402-0156

98. Stepanian A, Bourguignat L, Hennou S, et al. Microparticle increase in severe obesity: not related to metabolic syndrome and unchanged after massive weight loss. Obesity. 2013;21 (11):2236-2243. doi:10.1002/oby.20365

99. Murakami T, Horigome $\mathrm{H}$, Tanaka $\mathrm{K}$, et al. Impact of weight reduction on production of platelet-derived microparticles and fibrinolytic parameters in obesity. Thromb Res. 2007;119(1):45-53. doi:10.1016/j.thromres.20 05.12 .013

100. Mathieu M, Martin-Jaular L, Lavieu G, et al. Specificities of secretion and uptake of exosomes and other extracellular vesicles for cell-to-cell communication. Nat Cell Biol. 2019;21(1):9-17. doi:10.1038/s41556-018-0250-9

101. Chen Y, Liu W, Sun T, et al. 1, 25-Dihydroxyvitamin D promotes negative feedback regulation of TLR signaling via targeting microRNA-155-SOCS1 in macrophages. J Immunol. 2013;190 (7):3687-3695. doi:10.4049/jimmunol.1203273 
102. Ramon S, Dalli J, Sanger JM, et al. The protectin PCTR1 is produced by human M2 macrophages and enhances resolution of infectious inflammation. Am J Pathol. 2016;186(4):962-973. doi:10.1016/j.ajpath.2015.12.012

103. Bourlier V, Sengenès $C$, Zakaroff-Girard A, et al. TGF $\beta$ family members are key mediators in the induction of myofibroblast phenotype of human adipose tissue progenitor cells by macrophages. PLoS One. 2012;7(2):e31274. doi:10.1371/journal. pone. 0031274

104. Castaño C, Kalko S, Novials A, et al. Obesity-associated exosomal miRNAs modulate glucose and lipid metabolism in mice. Proc Natl Acad Sci USA. 2018;115(48):12158-12163. doi:10. 1073/pnas. 1808855115

105. Ying $\mathrm{H}$, Kang $\mathrm{Y}$, Zhang $\mathrm{H}$, et al. MiR-127 modulates macrophage polarization and promotes lung inflammation and injury by activating the JNK pathway. J Immunol. 2015;194(3):1239-1251. doi:10.4049/jimmunol.1402088

106. Xie T, Liang J, Liu N, et al. miRNA-127 inhibits lung inflammation by targeting IgG Fc $\gamma$ receptor I. J Immunol. 2012;188 (5):2437-2444. doi:10.4049/jimmunol.1101070

107. Zhao H, Shang Q, Pan Z, et al. Exosomes from adipose-derived stem cells attenuate adipose inflammation and obesity through polarizing M2 macrophages and beiging in white adipose tissue. Diabetes. 2018;67(2):235-247. doi:10.2337/db17-0356

108. Takanabe R, Ono K, Abe Y, et al. Up-regulated expression of microRNA-143 in association with obesity in adipose tissue of mice fed high-fat diet. BiochemBiophys Res Commun. 2008;376 (4):728-732. doi:10.1016/j.bbrc.2008.09.050

109. Jordan SD, Krüger M, Willmes DM, et al. Obesity-induced overexpression of miRNA-143 inhibits insulin-stimulated AKT activation and impairs glucose metabolism. Nat Cell Biol. 2011;13 (4):434-446. doi:10.1038/ncb2211
110. McGregor RA, Choi SM. microRNAs in the regulation of adipogenesis and obesity. Curr Mol Med. 2011;11(4):304-316. doi:10.2174/156652411795677990

111. Li $\mathrm{R}$, Shen $\mathrm{Q}, \mathrm{Wu} \mathrm{N}$, et al. MiR-145 improves macrophage-mediated inflammation through targeting Arf6. Endocrine. 2018;60(1):73-82. doi:10.1007/s12020-018-1521-8

112. Ma J, Wei K, Liu J, et al. Glycogen metabolism regulates macrophage-mediated acute inflammatory responses. Nat Commun. 2020;11(1):1769. doi:10.1038/s41467-020-15636-8

113. Talari M, Nayak TK, Kain V, et al. MicroRNA-712 restrains macrophage pro-inflammatory responses by targeting LRRK2 leading to restoration of insulin stimulated glucose uptake by myoblasts. Mol Immunol. 2017;82:1-9.

114. Liu T, Sun Y-C, Cheng P, et al. Adipose tissue macrophage-derived exosomal miR-29a regulates obesity-associated insulin resistance. Biochem Biophys Res Commun. 2019;515(2):352-358. doi:10.1016/j.bbrc.2019.05.113

115. Svensson H, Wetterling L, Andersson-Hall U, et al. Adipose tissue and body composition in women six years after gestational diabetes: factors associated with development of type 2 diabetes. Adipocyte. 2018;7(4):229-237. doi:10.1080/21623945.2018.15 21230

116. Neeland IJ, de Lemos JA. Time to retire the BMI?: Evaluating abdominal adipose tissue imaging as novel cardiovascular risk biomarker. J Am Coll Cardiol. 2016;68(14):1522-1524. doi:10. 1016/j.jacc.2016.03.613

117. Lesna IK, Cejkova S, Kralova A, et al. Human adipose tissue accumulation is associated with pro-inflammatory changes in subcutaneous rather than visceral adipose tissue. Nutr Diabetes. 2017;7(4):e264. doi:10.1038/nutd.2017.15

\section{Publish your work in this journal}

Diabetes, Metabolic Syndrome and Obesity: Targets and Therapy is an international, peer-reviewed open-access journal committed to the rapid publication of the latest laboratory and clinical findings in the fields of diabetes, metabolic syndrome and obesity research. Original research, review, case reports, hypothesis formation, expert opinion and commentaries are all considered for publication. The manuscript management system is completely online and includes a very quick and fair peer-review system, which is all easy to use. Visit http://www.dovepress.com/testimonials.php to read real quotes from published authors. 